\title{
Multi-Criteria Decision-Making Techniques for Solving the Airport Ground Handling Service Equipment Vendor Selection Problem
}

\author{
Chien-Wen Shen ${ }^{1}\left[\right.$, , Yen-Ting Peng ${ }^{1}$ and Chang-Shu Tu ${ }^{2,3,4, * \mathbb{C}}$ \\ 1 Department of Business Administration, National Central University, No.300, Jhongda Rd., Jhongli City \\ 32001, Taiwan; cwshen@ncu.edu.tw (C.-W.S.); amberpeng@hotmail.com (Y.-T.P.) \\ 2 Department of Information Management, Chang Gung University, Taiwan, ROC; 259 Wen-Hwa 1st Road, \\ Kwei-Shan, Tao-Yuan 33333, Taiwan \\ 3 Global Research \& Industry Alliance, Nation Taiwan University of Science, Taipei City 10607, Taiwan \\ 4 Taiwan Express Co., Ltd., Taipei City 1049, Taiwan \\ * Correspondence: long.tree@msa.hinet.net
}

Received: 28 May 2019; Accepted: 14 June 2019; Published: 24 June 2019

\begin{abstract}
Appropriate airport ground handling service (AGHS) equipment vendor selection (AGHSEVS) can prevent aircraft damage and delays in airlines schedules, and ensure reliable and high-quality ground handling service. Previous research has seldom integrated multi-criteria decision-making techniques with goal programming to solve the AGHSEVS problem. This paper describes a new system evaluation model for AGHSEVS by considering both qualitative and quantitative methods. We compare the fuzzy TOPSIS method based on fuzzy weighted average left and right score methods with multi-choice and multi-aspiration goal programming approach of an AGHS company in Taiwan. These study results can help airport ground handling service company managers make optimal decisions for AGHSEVS problems. We hope the practicability of the comparable model with slight modifications of real situation data can be used in other AGHS companies.
\end{abstract}

Keywords: equipment vendor selection; fuzzy TOPSIS; fuzzy weighted average left and right score; multi-choice goal programming; multi-aspiration goal programming

\section{Introduction}

A crucial task of airport ground handling service (AGHS) companies is to conduct diverse ground handling service processes on airport ramps. Two major types of ground handling service procedures are generally distinguished: terminal operations and ramp operations. In this paper, we focus on ramp operations because they require complex tasks and a diversity of AGHS equipment. As a service of AGHS companies, ramp handling (i.e., ground handling service) includes the loading and unloading of airplanes and the transport of passengers, crew, baggage, freight and mail between airplanes and terminal buildings. In general, this can be classified as an AGHS logistics service. This service is typically provided by a third-party ground handler (i.e., an AGHS company), the airline itself, or by the ramp handling business unit of an airport [1,2]. From the above description, we can know that the the AGHS company's main job is working on the ramp handling safely. However, selecting the most suitable equipment is difficult because characteristics vary. This makes the job of ground handlers for AGHS companies more difficult because equipment malfunctions can affect work safety on the airport ramps. Effective AGHS has benefits such as preventing aircraft damage (collisions with ground handling equipment) and delays, reducing the turnaround time of ground services and ground handling costs, and ensuring high handling reliability [3]. To avoid the aforementioned 
problem, AGHS companies rely on high-tech equipment to solve AGHS equipment vendor selection (AGHSEVS) problems. Most AGHS companies are concerned with equipment performance and quality. A numerous amount of AGHS equipment is available. We therefore compiled a list of generally required AGHS equipment, including aircraft towing trucks, main deck loaders, container pellet loaders, conveyor belt loaders, passenger step vehicles, ground power units, air starter units, catering trucks, and cargo towing tractors. Aircraft towing trucks are vital pieces of equipment. There are several types of aircraft towing trucks designed to push and tow aircraft. Aircraft towing trucks can tow aircraft weighting up to 100 metric tons [3]. Acquisition of an aircraft towing truck that satisfies operational performance, equipment quality, and ease of usage requirements is crucial to improving the operational safety of an AGHS company [4]. Hence, formulating a purchasing list of required AGHS equipment is regarded as both a decision-making problem and a reflection of company preference. AGHSEVS is a strategic problem that has a substantial effect on the safety of ramps work. Addressing AGHSEVS problems is a time-consuming and complex process that requires professional knowledge and experience for making judgments [5]. Thus, selecting an optimal equipment vendor is a multi-criteria decision making (MCDM) problem. The AGHSEVS process may be particularly complicated for AGHS company managers. Before an AGHS company purchases new equipment (such as a vehicle), it should analyze its finances and options because budget constraints can limit the types of equipment that can be purchased. Most AGHS companies rank safety high among their purchasing considerations when buying new equipment. Other important attributes include fuel economy, comfort, convenience features, insurance information, technical specifications, warranties, and resale value [6]. As the AGHS market becomes increasingly competitive, continual changes within AGHS companies demand that leading manufactures provide them with new and technologically superior equipment. Thus, an analytic decision-making method for selecting optimal AGHS equipment is useful to both the AGHS company and the equipment manufacturer. An analytic method not only reduces the buyer's burden but also may increase equipment sales. Additionally, it plays a strategic role by improving customer services within the competitive AGHS market environment. Although AGHSEVS is a crucial aspect of ramp operations, research on this subject is limited. A study by Bard and Sousk (1990) suggested the use of the analytic hierarchy process (AHP) to select the next generation of rough terrain cargo handlers for the U.S. Army [4]. Most relevant studies have employed MCDM methods to make equipment choices. Shang and Sueyoshi (1995) developed a decision support framework with three modules (AHP, simulation and accounting procedures) to assist managers in selecting the most appropriate flexible manufacturing system designs [7]. Lin and Yang (1996) recommended establishing a knowledge-based (i.e., expert system) model using the AHP for the selection of optimal machinery [8]. Chan et al. suggested an expert consultant system for the selection of material-handling equipment suitable for the movement and storage of materials in a manufacturing system [9]. Certain studies have proven particularly valuable for evaluating equipment, such as [10] who proposed a systematic evaluation model using AHP and fuzzy technique for order preference by similarity to ideal solution (TOPSIS) methods to help agents in defense industries select optimal weapons equipment among a set of available alternatives in a fuzzy environment. They utilized a MCDM method to determine the weight of evaluation criteria, and adopted a fuzzy TOPSIS method to obtain the performance ratings of feasible alternatives in linguistic values, parameterized using triangular fuzzy numbers. However, the fuzzy AHP process is impractical in some cases. Because of the numerous potential available choices and criteria [11,12], a fuzzy AHP decision approach may produce fatigue among DMs caused by repetitive measurements. Therefore, to avoid an unreasonably large number of pairwise comparisons, the fuzzy TOPSIS method is suitable for achieving an optimal ranking result. The aforementioned research offers a starting point. Regarding evaluation and selection problems, a decision-making model is required to select an optimal solution from the proposed alternatives [10]. However, previous research has rarely integrated the MCDM method with linear programming (LP) and goal programming (GP) approaches to solve the AGHSEVS problem. In reality, conflicting resources and incomplete information cause substantial difficulty for DMs in constructing a 
reliable mathematical model to illustrate their preferences [13]. To overcome this problem, numerous approaches have been developed and numerous methodologies have been applied. Generally, GP entails applying the logic of optimization in mathematical programming to satisfy several goals of the DMs [14]. For verifying an LP model, we employed multi-choice goal programming (MCGP) and multi-aspiration goal programming (MAGP) to perform comparative analyses.

The objective of this study was to help AGHS company managers adopt simple, systematic methods for obtaining high-quality equipment vendors. Additionally, we attempted to avoid the inherent drawbacks of using group decision-making systems to solve the AGHSEVS problem. We therefore incorporated experimental methods (AHP, fuzzy TOPSIS based on FWA left and right score, MCGP, and MAGP) for facilitating AGHSEVS problem to provide a reliable framework as a valuable reference for equipment buyers and suppliers. We adopt a new systematic evaluation procedure can help AGHS company managers make optimal decisions for AGHSEVS problems.

The remainder of this paper is structured as follows: Section 2 briefly describes our proposed method, Section 3 proposes and describes in detail a model for the AGHSEVS, and Section 4 presents a conclusion wherein management implications are discussed.

\section{Methods}

In this study, we employed three MCDM methods, namely the AHP method, the fuzzy TOPSIS method based on fuzzy weighted average (FWA) left and right score and LP and GP methods, namely MAGP and MCGP, to solve the AGHSEVS problem.

\subsection{AHP Method}

The AHP is a decision-support procedure developed by Saaty [15] for addressing complex, unstructured, multi-criteria decisions. The AHP is based on three factors: the structure of the model, the comparative evaluation of alternatives, and the criteria and synthesis of priorities. In the literature, the AHP has been widely used to solve numerous complicated decision-making problems $[10,16,17]$. In Step 1, a complex decision problem is structured as a hierarchy. The AHP initially deconstructs complex MCDM problems into a hierarchy of interrelated decision elements. Objectives, criteria, and alternatives are arranged in a hierarchical structure similar to a family tree. In Step 2, alternatives and criteria are compared. Once the problem has been deconstructed and a hierarchy has been formulated, a prioritization procedure for determining the relative importance of criteria within each level begins. A pairwise judgment starts at the second level and ends at the lowest level of alternatives. At each level, criteria are compared pairwise according to their levels of influence and on the basis of the specified criteria in higher levels [18].

In the AHP, multiple pairwise comparisons are based on a standardized comparison scale of nine levels (Table 1).

Table 1. Description of nine-point intensity of importance scale.

\begin{tabular}{cc}
\hline Intensity of Importance & Definition Meaning \\
\hline 1 & Equally important \\
3 & Moderately more important \\
5 & Strongly more important \\
7 & Very strongly more important \\
9 & Extremely more important \\
$2,4,6,8$ & Intermediate values \\
\hline
\end{tabular}

In Step 3, $C=\left\{C_{j} \mid j=1,2 \ldots, n\right\}$ represents the set of criteria. The result of the pairwise comparison of $n$ criteria can be summarized in an $(n \times n)$ evaluation matrix $D$ in which every element $d_{i j}(i, j=1,2$, $\ldots, n)$ is the quotient of weights of the criteria, as in Equation (1): 


$$
D=\left[\begin{array}{cccc}
d_{11} & d_{12} & \cdots & d_{1 n} \\
d_{21} & d_{22} & \cdots & d_{2 n} \\
\vdots & \vdots & \ddots & \vdots \\
d & d_{n 2} & \cdots & d_{n n}
\end{array}\right], d_{i i}=1, d_{j i}=1 / d_{i j}, d_{i j} \neq 0 .
$$

In Step 4, a mathematical process is used to normalize and calculate the relative weights for each matrix. Relative weights are determined by the eigenvector $(w)$ corresponding to the largest eigenvalue $\left(\lambda_{\max }\right)$ :

$$
D_{W}=\lambda_{\max } W .
$$

If the pairwise comparisons are completely consistent, the matrix $D$ has rank 1 , and $\lambda_{\max }=n$. Under this condition, weights can be obtained by normalizing any of the rows or columns of $D[5,10]$. In Step 5, the quality of the output of the AHP becomes strictly related to the consistency of the relation between the entries of $D$; that is, $d_{i j} \times d_{j k}=d_{i k}$. The consistency index $(C I)$ is

$$
C I=\frac{\left(\lambda_{\max }-n\right)}{(n-1)} .
$$

Step 6 entails delineating the final consistency ratio $(C R)$, which indicates whether the evaluations are sufficiently consistent. The final $C R$ is calculated as the ratio of $C I$ and the random index $(R I)$, as indicated in the following:

$$
C R=C I / R I \text {. }
$$

$R I$ should be lower than 0.10 to accept the AHP results as consistent. If the final $C R$ exceeds this value, the DM should return to Steps 2 and 3 and perform the assessments and comparison again. The consistency measurement can be used to evaluate the consistency of the overall hierarchy $[10,17,18]$.

\subsection{TOPSIS Method and Fuzzy TOPSIS Method}

TOPSIS was proposed by Hwang and Yoon [19]. The ideal solution, also termed the positive ideal solution, is a solution that maximizes beneficial criteria and attributes and minimizes cost criteria and attributes, whereas the negative ideal solution, also termed the anti-ideal solution, maximizes the cost criteria and attributes, and minimizes the benefit criteria and attributes. According to this technique, the optimal choice is the one that is closest to the ideal solution and farthest from the negative ideal solution. That is, the positive ideal solution is composed entirely of optimal values attainable from the criteria, whereas the negative ideal solution consists entirely of the worst values attainable from the criteria [20]. Numerous studies have employed TOPSIS to solve MCDM problems [21-23].

With the traditional formulation of TOPSIS, personal judgments are represented by crisp values. However, in many practical cases, human preferences are uncertain and DMs might be reluctant or unable to assign crisp values to the judgments $[10,24]$. The use of fuzzy set theory Zadeh enables DMs to incorporate unquantifiable information, incomplete information, and non-obtainable information into a decision model $[6,10,25]$. The fuzzy TOPSIS method can address ambiguities, uncertainties and vagueness in decision-making, which otherwise would be handled using crisp values. In its feasible applications, the triangular form of the membership function is frequently used to represent fuzzy numbers $[10,25-28]$. In the following subsection, the basic definitions and notation of fuzzy sets are provided and are used hereafter unless otherwise stated [29]. Our study uses a fuzzy TOPSIS method that performs the calculation process based on intervals of fuzzy ratings of alternatives and fuzzy weights. In order to eliminate this drawback and achieve the accurate results with the low computational volume, we apply FWA left and right scores instead of applying the alpha level sets of fuzzy numbers. 


\subsection{FWA Left and Right Scores}

In this study, the FWA left and right scores were utilized to select optimal AGHS equipment [30]. The FWA algorithm based on left and right scores is provided as follows:

Step 1: Set the linguistic variables for the importance of criteria and rating of alternatives, represented by fuzzy numbers.

Step 2: Evaluate the importance of criteria on the basis of the linguistic variables provided in Step 1.

Step 3: Let the fuzzy numbers $A=\left(a_{1}, b_{1}, c_{1}\right), B=\left(a_{2}, b_{2}, c_{2}\right)$ and $C=\left(a_{3}, b_{3}, c_{3}\right)$. Aggregate the fuzzy numbers as follows:

$$
A F N=\left(\frac{\left(a_{1}+b_{1}+c_{1}\right)}{3}, \frac{\left(a_{2}+b_{2}+c_{2}\right)}{3}, \frac{\left(a_{3}+b_{3}+c_{3}\right)}{3}\right) .
$$

Step 4: Normalize the fuzzy decision matrix to determine cost and benefit criteria:

$$
\begin{aligned}
\left(\widetilde{y}_{i j}\right)_{N} & =\left(\left(a_{i j}\right)_{N^{\prime}}\left(b_{i j}\right)_{N^{\prime}}\left(c_{i j}\right)_{N}\right) \\
& =\left[\frac{a_{i j}-a_{j}^{M i n}}{\Delta_{\text {Min }}^{\text {Max }}}, \frac{b_{i j}-a_{j}^{\text {Min }}}{\Delta_{\text {Min }}^{\text {Max }}}, \frac{c_{i j}-a_{j}^{\text {Min }}}{\Delta_{\text {Min }}^{\text {Max }}}\right], j=1, \ldots, n ; j \in \Omega_{b} \\
\left(\widetilde{y}_{i j}\right)_{N} & =\left(\left(a_{i j}\right)_{N^{\prime}}\left(b_{i j}\right)_{N^{\prime}}\left(c_{i j}\right)_{N}\right) \\
& =\left[\frac{a_{i j}-a_{j}^{\text {Max }}}{\Delta_{\text {Max }}^{\text {Min }}}, \frac{b_{i j}-a_{j}^{\text {Max }}}{\Delta_{\text {Max }}^{\text {Min }}}, \frac{c_{i j}-a_{j}^{\text {Max }}}{\Delta_{\text {Max }}^{\text {Min }}}\right], j=1, \ldots, n ; j \in \Omega_{c},
\end{aligned}
$$

where

$\left(a_{i j}, b_{i j}, c_{i j}\right)_{N}=$ normalized fuzzy number,

$c_{j}^{\operatorname{Max}}=\operatorname{Max} c_{i j}, c_{j}^{\text {Min }}=\operatorname{Min} a_{i j}, j=1, \ldots, n$,

$\Delta_{\text {Min }}^{\text {Max }}=c_{\text {Min }}^{\text {Max }}-a_{j}^{\text {Min }}, \Delta_{\text {Max }}^{\text {Min }}=c_{j}^{M i n}-a_{j}^{\text {Max }}$,

$\Omega_{b}=$ set of benefit criteria,

$\Omega_{c}=$ set of cost criteria.

Step 5: Calculate the left $\left(L_{s}\right)_{i j}$ and right scores $\left(R_{S}\right)_{i j}$ by using the following formulas:

$$
\left(L_{s}\right)_{i j}=\frac{\left(b_{i j}\right)_{N}}{1+\left(b_{i j}\right)_{N}-\left(a_{i j}\right)_{N}} \text { and }\left(R_{s}\right)_{i j}=\frac{\left(c_{i j}\right)_{N}}{1+\left(c_{i j}\right)_{N}-\left(b_{i j}\right)_{N}} .
$$

Step 6: Evaluate the alternatives with respect to the criteria on the basis of the linguistic variables provided in Step 1.

Step 7: Determine the average fuzzy number, normalized fuzzy weights and left and right scores of alternatives with respect to the criteria by repeating Steps 3, 4 and 5 .

Step 8: Calculate the value of the FWA by integrating the left and right scores for criteria and alternatives simultaneously:

$$
\text { FWA, } \theta_{i}=\frac{w_{1} r_{i 1}+w_{2} r_{i 2}+\ldots+w_{m} r_{m}}{w_{1}+w_{2}+\ldots+w_{m}}=\frac{\sum_{j=1}^{m}\left(w_{j} \times r_{i j}\right)}{\sum_{j=1}^{m} w_{j}},
$$

where

$w_{j}=$ left and right scores of criteria,

$r_{i j}=$ left and right scores of alternatives. 
Step 9: Calculate the average of FWA value $\left(\left(\theta_{i}\right)\right)_{\text {Average }}$, for each alternative using:

$$
\left(\left(\theta_{i}\right)\right)_{\text {Average }}=\frac{\left(\theta_{i}\right)^{U}+\left(\theta_{i}\right)^{L}}{2}, j=1, \ldots, n,
$$

where

$\left(\theta_{i}\right)_{U}=$ the upper interval for each alternative,

$\left(\theta_{i}\right)_{L}=$ the lower interval for each alternative.

Step 10: Rank the alternatives according to the average values in descending order to obtain the final results.

\section{Three Models of AGHSEVS}

\subsection{LP Model for AGHSEVS}

In current complex organizations, DMs do not attempt to maximize a well- defined utility function. Conflicts of interest and the incompleteness of available information make it nearly impossible to construct a reliable mathematical representation of DM preferences. Utilizing these deviational variables, we adopted the LP model proposed by Guneri et al. [31].

The objective function and constraints of the LP approach for AGHSEVS are as follows:

Nomenclature Variable

$C C_{i}$ closeness coefficients of $i$ th vendor,

$X_{i}$ order quantity for $i$ th vendor,

$D$ total demand (30 units in the model),

$q_{i} \quad$ defect quality rate of $i$ th vendor,

$Q$ company's maximum acceptable defect quality rate (0.04 in the model),

$P_{i} \quad$ unit price of $i$ th vendor,

$P \quad$ company's maximum acceptable unit price with respect to the allocated budget for purchasing the order (9.2 million US dollar in the model),

$C_{i}$ capacity of $i$ th vendor,

$$
\operatorname{Max}(\mathrm{TVP})=\sum_{i=1}^{n} C C_{i} X_{i} \text { (objective function), }
$$

Subject to

$$
\begin{gathered}
\sum_{i=1}^{n} X_{i}=D \text { (demand constraint), } \\
\sum_{i=1}^{n} X_{i} p_{i} \leq P D(\text { purchase cos t budgeting constraint), } \\
\sum_{i=1}^{n} X_{i} q_{i} \leq Q D \text { (AGHS equipment quality constraint), } \\
X_{i} \leq C_{i}(\text { vendor capacity constraint), } \\
X_{i} \leq 0, i=1,2, \ldots, n \text { (non-negativity constraint). }
\end{gathered}
$$

Equation (5) is the objective function of total value purchasing (TVP), Equation (6) represents the demand constraint of the AGHS company, Equation (7) represents the budgeting constraint of the AGHS company, Equation (8) represents the equipment quality constraint of each vendor, Equation (9) 
represents the capacity constraint of each vendor, and Equation (10) represents the non-negativity constraint which prohibits negative orders.

\subsection{MCGP Model for AGHSEVS}

GP, proposed by Charnes, Cooper, and Ferguson (1955) [32], is the most widely used approach in MCDM [33]. It is designed to address problems involving multiple conflicting objectives [34]. The MCGP approach includes many modified GP methods conceived by previous scholars. To improve the utility of the GP techniques, Chang developed a multi-choice aspiration level (MCAL) model for solving multi-objectives decision-making (MODM) problems [35]. Chang's proposed approach differs considerably from fuzzy GP because his model incorporates membership functions for addressing MODM problems with imprecise goal aspiration levels. This decision-making method can be used to set various aspiration levels, and can sort solution strategies (e.g., the higher the aspiration level achieved, the more favorable the outcome). The MCAL model can be employed to determine the most suitable resources for achieving higher aspiration levels at the initial stage of the resolution process. A typical MCGP problem is described as follows.

In real decision-making problems, goals are often interrelated. This problem is addressed in the following MCGP equations:

$$
\operatorname{Minimize} \sum_{i=1}^{n}\left[\left(d_{i}^{+}+d_{i}^{-}\right)+\left(e_{i}^{+}+e_{i}^{-}\right)\right]
$$

Subject to

$$
\begin{array}{cc}
f_{i}(X) b_{i}-d_{i}^{+}+d_{i}^{-}=b_{i} y_{i} & i=1,2, \ldots, n, \\
y_{i}-e_{i}^{+}+e_{i}^{-}=g_{i, \min } & i=1,2, \ldots, n, \\
g_{i, \min } \leq y_{i} \leq g_{i, \max } & i=1,2, \ldots, n \\
d_{i}^{+}, d_{i}^{-}, e_{i}^{+}, e_{i}^{-} \geq 0, & i=1,2, \ldots, n .
\end{array}
$$

As illustrated in Equations (12), (13), and (14), there are no selection restrictions for a single goal, but some dependent relationships exist among the goals. For instance, we can add the auxiliary constraint $b_{i} \leq b_{i+1}+b_{i+2}$ to the MCGP model, where $b_{i}, b_{i+1}$ and $b_{i+2}$ are binary variables. Consequently, $b_{i+1}$ or $b_{i+2}$ must equal 1 if $b_{i}=1$. This means that, if goal 1 has been achieved, then either goal 2 or goal 3 has correspondingly been achieved.

\subsection{MAGP Model for AGHSEVS}

Hossein and Attarpour integrated MAGP and MCGP to solve multi-aspiration problems [36]. For comparison with the MCGP model, we adopted an MAGP model for AGHSEVS. In this section, an AGHS company's usage of MAGP is introduced. Aircraft towing truck vendor are classified into five types $\left(X_{1} \ldots X_{5}\right)$ in five manufacturing areas (i.e., $X_{1}$ : Hong Kong, $X_{2}$ : Japan, $X_{3}$ : United States, $X_{4}$ : France and $X_{5}$ : Germany), which serve as manufacturer market segments in this real case. The AGHS company managers seek to achieve four predetermined goals. These goals are Goal $1\left(g_{1}\right)$ the TVP of at least 20 units from procurement; Goal $2\left(g_{2}\right)$ a total procurement cost less than 288 million US dollars; Goal $3\left(g_{3}\right)$ a minimal rate of defective machinery (less than 0.04$)$ received from the vendor; and Goal $4\left(g_{4}\right)$, for implementing a differentiation strategy (i.e., quality leadership), maintaining a current procurement level of fewer than 35 units. The quantity for these goals covers two choices: one or a proportion of both can be accepted. The AGHS company manager must determine the correct model and quantity of an aircraft towing truck to achieve the optimal result. Importing aircraft towing trucks entails expenses. To economize, at least $D=30$ units of the aircraft towing truck should be selected. Consequently, certain constraints are added to the model to guarantee that these conditions are satisfied. Table 2 lists the coefficient and goal values for solving the MAGP problem. 
Table 2. Cofficient and goal values for the multi-aspiration goal programming approach model.

\begin{tabular}{ccccccc}
\hline \multicolumn{7}{c}{ Coefficient Segments of Variable } \\
\hline $\boldsymbol{X}_{\mathbf{1}}$ & $\boldsymbol{X}_{\mathbf{2}}$ & $\boldsymbol{X}_{\mathbf{3}}$ & $\boldsymbol{X}_{\mathbf{4}}$ & $\boldsymbol{X}_{\mathbf{5}}$ & Choice Value & Goal \\
\hline 0.362 & 0.35 & 0.37 & 0.324 & 0.340 & 20,30 & Total value \\
7.9 & 8.9 & 9.3 & 9.5 & 9.6 & 276,288 & Cost(Price) \\
0.05 & 0.04 & 0.06 & 0.01 & 0.02 & $1.2,1.8$ & Delivery defect rate \\
1 & 1 & 1 & 1 & 1 & 30,35 & Procurement level \\
Hong Kong & Japan & United States & France & Germany & & Manufacture areas \\
\hline
\end{tabular}

The MAGP model's notation is introduced as follows:

Indices:

i for goals,

$l$ for aspiration levels,

$k$ for segments,

j for decision variables,

Variables:

$x_{j}$ decision variable,

$x_{j k}$ part of the $j$ th decision variable in the $k$ th segment,

$s_{j k} \quad$ coefficient for the $j$ th decision variable and the $k$ th segment,

$Z_{l} \quad$ coefficient for the $l$ th aspiration level,

$d_{i}^{+}$positive deviation from the $i$ th goal target value,

$d_{i}^{-} \quad$ negative deviation from the $i$ th goal target value,

Parameters:

$n$ number of goals,

$m$ number of decision variables,

$u$ number of aspiration levels for goal,

$h_{j}$ number of segments for the $j$ th decision variable,

C a constant that is related to the DM,

Hence, the MAGP model can be expressed as follows:

$$
\operatorname{Minimize} \sum_{i=1}^{n}\left(d_{i}^{+}+d_{i}^{-}\right) \text {, }
$$

Subject to

$$
\begin{gathered}
\sum_{j=1}^{m} \sum_{k=1}^{h_{j}} S_{i j k}-d_{i}^{+}+d_{i}^{-}=\sum_{i=1}^{u} z_{l} g_{i l}, i=1,2, \ldots, n \\
\sum_{k=1}^{h_{j}} x_{j k}=x_{j}, j=1,2, \ldots, m \\
\sum_{j=1}^{m} x_{j k}=C, k=1,2, \ldots, h_{j} \\
\sum_{j=1}^{u} z_{l}=1, \\
d_{i}^{+}, d_{i}^{-} \geq 0, i=1,2, \ldots, n
\end{gathered}
$$




\subsection{Solution Procedure}

AGHSEVS is a problem involving multi-criteria, alternatives and evaluators; we developed a model for AGHSEVS in this study. The proposed model was designed on the basis of comparisons among AGHS equipment alternatives according to identified criteria. The AHP and fuzzy TOPSIS methods are used in the proposed model. The proposed model differs from equipment selection processes described in the relevant literature. The AHP is used to assign weights to the criteria to be used for AGHSEVS, and fuzzy TOPSIS is employed to prioritize the alternatives. The weights obtained from the AHP are included in the decision-making process by incorporating them into fuzzy TOPSIS computations, and the alternative priorities are determined by these weights. Thus, the weighting of criteria considered during decision-making and the evaluation of these criteria are performed simultaneously. Our proposed model substantially increases the efficiency of the decision-making process for AGHSEVS.

In Stage 1, criteria that will be used in the evaluation are determined and the decision hierarchy is formed. Following the approval of the decision hierarchy, the criterion weights are assigned by an expert AGHSEVS decision-making team in Stage 2, wherein pairwise comparison matrices are formed. The team makes individual evaluations by using the scale provided in Table 1, to determine the elements values of pairwise comparison matrices. AGHSEVS evaluation criteria ranks are determined using the fuzzy TOPSIS method based on fuzzy weighted average left and right score in Stage 3. Linguistic values are used for the evaluation of AGHSEVS in this step. The membership functions of these linguistic values are displayed in Figure 1, and the triangular fuzzy numbers associated with these variables are displayed in Table 3. In Stage 4, we use the AGHSEVS LP model with two GP approaches for the AGHSEVS problem. Figure 2 illustrates the proposed method for AGHSEVS problem, comprising Stages 1 to 4 (Figure 2).

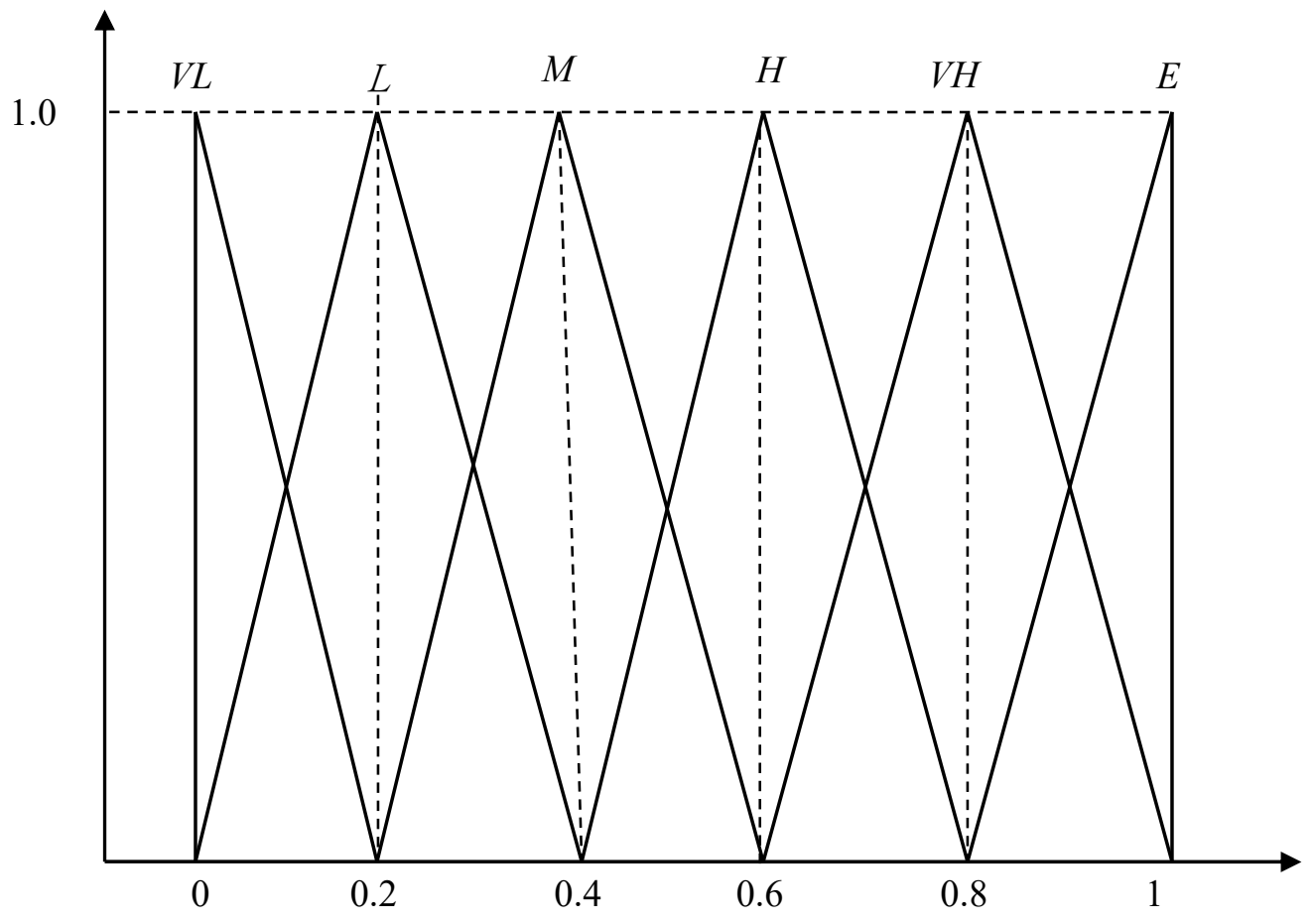

Figure 1. Membership functions of linguistic values for criteria. 
Table 3. Linguistic values and associated fuzzy numbers.

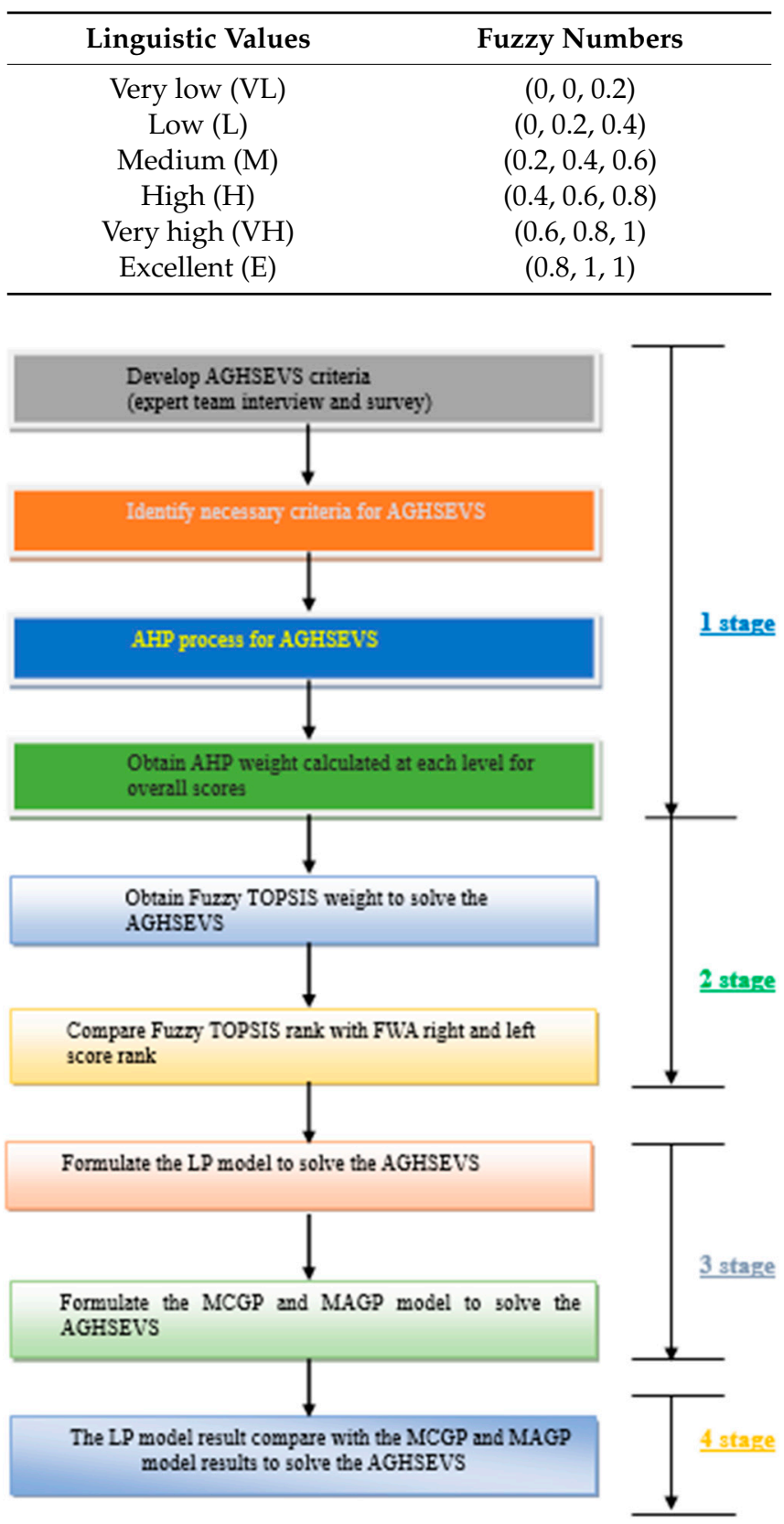

Figure 2. Schematic diagram of the proposed model for airport ground handling service vendor selection problem.

According to the previously summarized fuzzy theory (Stages 1-3), the fuzzy TOPSIS steps can be outlined [10,31]. In Stage 4, the LP, MCGP, and MAGP approaches are applied for AGHSEVS and the computational procedure is summarized as follows:

Step 1: Develop AGHSEVS criteria (expert team interview and survey).

Step 2: Identify necessary criteria for AGHSEVS and obtain AHP weight calculated at each level for overall scores.

Step 3: Select the linguistic values $\left(x_{i j}, i=1,2, \ldots, n, J=1,2, \ldots, k\right)$ for alternatives with respect to criteria. The fuzzy linguistic rating $\left(x_{i j}\right)$ preserves the property of ranges of normalized triangular fuzzy numbers belonging to $[0,1]$; thus, normalization is not required.

Step 4: Calculate the weighted normalized fuzzy decision matrix. 
Step 5: Identify positive-ideal $\left(A^{+}\right)$and negative ideal $\left(A^{-}\right)$solutions. These solutions are provided in the following equations:

$$
\begin{aligned}
& \mathrm{A}^{+}=\left\{\widetilde{\mathrm{v}}_{1}^{+}, \widetilde{\mathrm{v}}_{2}^{+}, \ldots \widetilde{\mathrm{v}}_{\mathrm{i}}^{+}\right\}=\left\{\left(\max _{j} v_{i j} \mid i \in I^{\prime}\right) \times\left(\min _{j} v_{i j} \mid i \in I^{\prime \prime}\right)\right\}, i=1,2, \ldots, n j=1,2, \ldots, k ; \\
& \mathrm{A}^{+}=\left\{\widetilde{\mathrm{v}}_{1}^{+}, \widetilde{\mathrm{v}}_{2}^{+}, \ldots \widetilde{\mathrm{v}}_{\mathrm{i}}^{+}\right\}=\left\{\left(\min _{j} v_{i j} \mid i \in I^{\prime}\right) \times\left(\max _{j} v_{i j} \mid i \in I^{\prime \prime}\right)\right\}, i=1,2, \ldots, n j=1,2, \ldots, k,
\end{aligned}
$$

where $I^{\prime}$ is associated with benefit criteria and $I^{\prime \prime}$ is associated with cost criteria.

Step 6: Calculate the distance of each alternative from $A^{+}$and $A^{-}$by using the following equations:

$$
\begin{aligned}
& D_{j}^{+}=\sum_{j=1}^{n} d\left(\widetilde{v}_{i j}, v_{i}^{+}\right) j=1,2 \ldots J, \\
& D_{j}^{-}=\sum_{j=1}^{n} d\left(\widetilde{v}_{i j}, v_{i}^{-}\right) j=1,2 \ldots J .
\end{aligned}
$$

Step 7: Calculate similarities to the ideal solution:

$$
C C_{j}=\frac{D_{j}^{-}}{D_{j}^{+}+D_{j}^{-}} j=1,2 \ldots J .
$$

Step 8: Rank the preference order. Choose an alternative with maximum $\mathrm{CC}_{j}^{+}$or rank alternatives according to $\mathrm{CC}_{j}^{+}$in descending order. An alternative with the index $C C_{j}$ approaching 1 indicates that the alternative is close to the fuzzy positive ideal reference point and far from the fuzzy negative ideal reference point. Note that a large value of the index $C C_{j}$ indicates the favorable performance of an alternative $A_{j}[5]$.

Step 9: Compare fuzzy TOPSIS and FWA left and right scores results.

Step 10: According to the closeness coefficients (Table 10) obtained from Step 8, build a LP model to determine the ideal vendors and their optimal order quantities. To select the optimal order quantities, the TVP should be maximized [36].

Step 11: According to the closeness indices obtained from Step 8, the LP method can be expressed as Equation (27) to solve the AGHSEVS problem as follows:

$$
\begin{aligned}
& \operatorname{Max}(\text { TVP })=0.362 X_{1}+0.350 X_{2}+0.370 X_{3}+0.324 X_{4}+0.340 X_{5}, \\
& 0.362 X_{1}+0.350 X_{2}+0.370 X_{3}+0.324 X_{4}+0.340 X_{5}<=30, \\
& 7.9 X_{1}+8.9 X_{2}+9.3 X_{3}+9.5 X_{4}+9.6 X_{5}<=288, \\
& 5 X_{1}+4 X_{2}+6 X_{3}+X_{4}+2 X_{5}<=180, \\
& X_{1}+X_{2}+X_{3}+X_{4}+X_{5}<=35, \\
& X_{1}<=15 ; \text { (vendor } E A_{1} \text { capacity constraint), } \\
& X_{2}<=10 ; \text { (vendor } E A_{2} \text { capacity constraint), } \\
& X_{3}<=20 ; \text { (vendor } E A_{3} \text { capacity constraint) } \\
& X_{4}<=30 ; \text { (vendor } E A_{4} \text { capacity constraint) }
\end{aligned}
$$




$$
\begin{gathered}
X_{5}<=12 ; \quad \text { (vendor } E A_{5} \text { capacity constraint), } \\
X_{1}>=0 ; X_{2}>=0 ; X_{3}>=0 ; X_{4}>=0 ; X_{5}>=0 .
\end{gathered}
$$

To solve an AGHSEVS problem, it is necessary to define the MCGP model according to the following goals. The manager of the AGHS company must determine the ideal vendors and their corresponding optimal quantities. According to sales records for the previous five years and sales forecasts for the company, the CEO and top managers of the company must establish four goals: Goal 1 $\left(g_{1}\right)$, a TVP of at least 20 units from procurement (the more the better); Goal $2\left(g_{2}\right)$, a total procurement cost less than 288 million US dollars (the less the better); Goal $3\left(g_{3}\right)$, for achieved the procurement levels, a minimal rate of defective machinery (less than 0.04 ) received from the vendors (the less the better); Goal $4\left(g_{4}\right)$, for implementing a differentiation strategy (i.e., quality leadership), a current procurement level of less than 35 units (the more the better). Variable coefficients in the model were calculated using a database comprising records of the preceding five years. The unit costs for the vendors $E A_{i}(i=1, \ldots, 5)$ are $7.9,8.9,9.3,9.5$ and 9.6 million US dollars. According to the historical data, the rate of defective machinery from vendors $E A_{i}(i=1, \ldots, 5)$ are $0.05,0.04,0.06,0.01$, and 0.02; delivery defects are set between 0.02 and 0.04 , and the capacities of the five candidate vendors $E A_{i}$ $(i=1, \ldots, 5)$ are $15,10,20,30$, and 12 units. The functions and parameters related to the AGHSEVS problem are listed as follows:

$f_{1}(x)=0.362 X_{1}+0.350 X_{2}+0.370 X_{3}+0.324 X_{4}+0.340 X_{5} \geq 20$ and $\leq 30\left(g_{1}:\right.$ TVP goal, the more the better),

$f_{2}(x)=7.9 X_{1}+8.9 X_{2}+9.3 X_{3}+9.5 X_{4}+9.6 X_{5} \geq 276$ and $\leq 288$ ( $g_{2}$ : cost goal, the less the better; i.e., $\$ 7.9 \times 30$ units $=\$ 276 ; \$ 9.6 \times 30$ units $=\$ 288)$,

$f_{3}(x)=0.05 X_{1}+0.04 X_{2}+0.06 X_{3}+0.01 X_{4}+0.02 X_{5}<=1.8\left(g_{3}\right.$ : delivery defect rate goal, the less the better; i.e., $0.06 \times 30$ units $=1.8$ ),

$f_{4}(x)=X_{1}+X_{2}+X_{3}+X_{4}+X_{5} \geq 30$ and $\leq 35$ ( $g_{4}$ : procurement level goal, the more the better),

$\operatorname{Max}(\mathrm{TVP})=0.362 X_{1}+0.350 X_{2}+0.370 X_{3}+0.324 X_{4}+0.340 X_{5}$

Subject to:

$X_{1}+X_{2}+X_{3}+X_{4}+X_{5}=30$ (demand constraint),

$5 X_{1}+4 X_{2}+6 X_{3}+X_{4}+2 X_{5}<=180$ (equipment quality constraint),

$7.9 X_{1}+8.9 X_{2}+9.3 X_{3}+9.5 X_{4}+9.6 X_{5}<=288$ (budgeting constraint),

$$
\begin{aligned}
& X_{1}<=15 \text { (vendor } E A_{1} \text { capacity constraint), } \\
& X_{2}<=10 \text { (vndor } E A_{2} \text { capacity constraint), } \\
& X_{3}<=20 \text { (vendor } E A_{3} \text { capacity constraint), } \\
& X_{4}<=30 \text { (vendor } E A_{4} \text { capacity constraint), } \\
& X_{5}<=12 \text { (vendor } E A_{5} \text { capacity constraint). }
\end{aligned}
$$

The following notation is defined to formulate the model:

Indices:

i $1,2, \ldots, n$ index of vendors,

$j \quad 1,2, \ldots, J$ index of deviation corresponding to the goals,

$t \quad 1,2, \ldots, T$ index of deviation corresponding to the multiple criteria,

Parameters:

$C_{i} \quad$ cost of material of vendor $i$,

$O_{i} \quad$ order cost of vendor $i$. 
$C C_{i} \quad$ closeness coefficient of vendor $i$,

$V \quad$ value of purchasing budget,

$d_{j}^{+}, d_{j}^{-}$maximum and minimum deviation of goal $j$,

$e_{t}^{+}, e_{t}^{-}$maximum and minimum deviation of $\left|y_{t}-g_{i, \max / \min }\right|$,

$q_{i} \quad$ rate of delivery defects of vendor $i$,

$p_{i} \quad$ rate of delivery delay number of vendor $i$,

$Q \quad$ maximum acceptable rate of delivery defects,

$P \quad$ maximum acceptable rate of delivery delay number,

D demand,

$S_{i} \quad$ capacity of vendor $i$,

Decision variables:

$X_{i} \quad$ order quantity of vendor $i$

$y_{\mathrm{i}} \quad$ binary integer $\begin{cases}1 & \text { if the order is of fered by vendors } \\ 0 & \text { otherwise }\end{cases}$

According to the closeness indices obtained from Step 6, the MCGP method can be expressed as Equation (29) to select the optimal equipment vendor.

Min $d_{1}^{+}+d_{1}^{-}+d_{2}^{+}+d_{2}^{-}+d_{3}^{+}+d_{3}^{-}+e_{1}^{+}+e_{1}^{-}+e_{2}^{+}+e_{2}^{-}+e_{3}^{+}+e_{3}^{-}$ TVP $=0.362 X_{1}+0.350 X_{2}+0.370 X_{3}+0.324 X_{4}+0.340 X_{5}$

$$
\begin{aligned}
& f_{1}=0.362 X_{1}+0.350 X_{2}+0.370 X_{3}+0.324 X_{4}+0.340 X_{5} \\
& f_{1}>=20 ; f_{1}<=30 \\
& f_{2}=7.9 X_{1}+8.9 X_{2}+9.3 X_{3}+9.5 X_{4}+9.6 X_{5} \\
& f_{2}>=276 ; f_{2}<=288 \\
& f_{3}=0.05 X_{1}+0.04 X_{2}+0.06 X_{3}+0.01 X_{4}+0.02 X \\
& f_{3}>=1.2 ; f_{3}<=1.8 \\
& f_{4}=X_{1}+X_{2}+X_{3}+X_{4}+X_{5} \\
& f_{4}>=30 ; f_{4}<=35 \\
& \left(0.362 X_{1}+0.350 X_{2}+0.370 X_{3}+0.324 X_{4}+0.340 X_{5}\right) b_{1}-d_{1}^{+}+d_{1}^{-}=\mathrm{y}_{1} b_{1} \\
& y_{1}-e_{1}^{+}+e_{1}^{-}=20 \\
& y_{1}>=20 \\
& y_{1}<=35 \\
& \left(7.9 X_{1}+8.9 X_{2}+9.3 X_{3}+9.5 X_{4}+9.6 X_{5}\right) b_{2}-d_{2}^{+}+d_{2}^{-}=y_{2} b_{2} \\
& y_{2}>=276 \\
& y_{2}<=288 \\
& \left(0.05 X_{1}+0.04 X_{2}+0.06 X_{3}+0.01 X_{4}+0.02 X_{5}\right) b_{3}-d_{3}^{+}+d_{3}^{-}=y_{3} b_{3} \\
& y_{3}-e_{2}^{+}+e_{2}^{-}=1.2 \\
& y_{3}>=1.2 \\
& y_{3}<=1.8 \\
& \left(X_{1}+X_{2}+X_{3}+X_{4}+X_{5}\right) b_{4}-d_{4}^{+}+d_{4}^{-}=y_{4} b_{4} \\
& y_{4}-e_{3}^{+}+e_{3}^{-}=30 \\
& y_{4}>=30 \\
& y_{4}<=35 \\
& b_{1}=b_{2}+b_{3}+b_{4}
\end{aligned}
$$


$b_{1}+b_{2}+b_{3}+b_{4}=1$

$X_{1}<=15$

$X_{2}<=10$

$X_{3}<=20$

$X_{4}<=30$

$X_{5}<=12$

$X_{1}>=0 ; X_{2}>=0 ; X_{3}>=0 ; X_{4}>=0 ; X_{5}>=0$;

$d_{1}^{+}>=0 ; d_{1}^{-}>=0 ; d_{2}^{+}>=0 ; d_{2}^{-}>=0 ; d_{3}^{+}>=0 ; d_{3}^{-}>=0 ; d_{4}^{+}>=0 ; d_{4}^{-}>=0$

$e_{1}^{+}>=0 ; e_{1}^{-}>=0 ; e_{2}^{+}>=0 ; e_{2}^{-}>=0 . e_{3}^{+}>=0 ; e_{2}^{-}>=0$.

Step 12: Using the MCGP method can be expressed as Equations (28) and (29) to select the optimal equipment vendor.

Step 13: Using the MAGP method can be expressed as Equation (30) to select the optimal equipment vendor.

$$
\begin{aligned}
& \operatorname{Min} d_{1}^{+}+d_{1}^{-}+d_{2}^{+}+d_{2}^{-}+d_{3}^{+}+d_{3}^{-} \\
& \text {TVP }=0.362 X_{1}+0.350 X_{2}+0.370 X_{3}+0.324 X_{4}+0.340 X_{5} \\
& f_{1}=0.362 X_{1}+0.350 X_{2}+0.370 X_{3}+0.324 X_{4}+0.340 X_{5} \\
& f_{2}=7.9 X_{1}+8.9 X_{2}+9.3 X_{3}+9.5 X_{4}+9.6 X_{5} \\
& f_{3}=0.05 X_{1}+0.04 X_{2}+0.06 X_{3}+0.01 X_{4}+0.02 X_{5} \\
& f_{4}=X_{1}+X_{2}+X_{3}+X_{4}+X_{5} \\
& 0.362 X_{1}+0.350 X_{2}+0.370 X_{3}+0.324 X_{4}+0.340 X_{5}-d_{1}^{+}+d_{1}^{-}=20 Z_{1}+30 Z_{2} \\
& 7.9 X_{1}+8.9 X_{2}+9.3 X_{3}+9.5 X_{4}+9.6 X_{5}-d_{2}^{+}+d_{2}^{-}=276 Z_{1}+288 Z_{2} \\
& 0.05 X_{1}+0.04 X_{2}+0.06 X_{3}+0.01 X_{4}+0.02 X_{5}-d_{3}^{+}+d_{3}^{-}=120 Z_{1}+180 Z_{2} \\
& X_{1}+X_{2}+X_{3}+X_{4}+X_{5}-d_{4}^{+}+d_{4}^{-}=30 Z_{1}+35 Z_{2} \\
& X_{1}<=15 \text { (vendor } E A_{1} \text { capacity constraint) } \\
& X_{2}<=10 \text { (vendor } E A_{2} \text { capacity constraint) } \\
& X_{3}<=20 \text { (vendor } E A_{3} \text { capacity constraint) } \\
& X_{4}<=30 \text { (vendor } E A_{4} \text { capacity constraint) } \\
& X_{5}<=12 \text { (vendor } E A_{5} \text { capacity constraint) } \\
& Z_{1}+Z_{2}=1 \\
& Z_{1}>=0 ; Z_{2}>=0 \\
& X_{1}>=0 ; X_{2}>=0 ; X_{3}>=0 ; X_{4}>=0 ; X_{5}>=0 \\
& d_{1}^{+}>=0 ; d_{1}^{-}>=0 ; d_{2}^{+}>=0 ; d_{2}^{-}>=0 ; d_{3}^{+}>=0 ; d_{3}^{-}>=0 ; d_{4}^{+}>=0 ; d_{4}^{-}>=0 .
\end{aligned}
$$

Step 14: Compare the results of two GP models for AGHSEVS.

\section{Case Application}

The objective of this section is to illustrate the model by using a realistic application for an AGHS company-Taoyuan International Airport Services Co., Ltd. (TIAS), which is a joint-venture ground handling service company owned by China Airlines (Taiwan) (49\%), the Ministry of Transportation and Communications (45\%), and the United Parcel Service (Taiwan branch) (6\%). TIAS offers a full 
range of handling services for all airlines and air cargo forwarders at Taoyuan Airport. TIAS is the first ground handler in Taiwan to acquire ISO 9001 accreditation from an internationally recognized quality management systems aimed at maintaining high-quality service standards. In addition, TIAS, as a member of the International Air Transport Association Ground Handling Council, keeps pace with international ground handling industry developments and is committed to providing superior ground handling services. In 2013, Taoyuan airport provided ground handling services for 194,239 flights, 30,701,987 passengers, and 1,571,814,282 tons of cargo. Currently, TIAS has 1028 units of vehicular and 4,658 units of non-vehicular ground handling equipment.

The aim of this research was to evaluate alternative AGHSEVS problems and to help the DMs at AGHS companies meet purchasing requirements. However, it is difficult to select the most suitable equipment because characteristics vary. For a real-world application, an expert AGHSEVS decision-making team was formed comprising two airport ground handling service department vice presidents and four senior technical supply and maintenance managers at TIAS. All criteria used in the model were determined in advance by the decision-making team for obtaining criterion weights.

\subsection{Identification of Criteria for AGHSEVS}

Criteria considered in the selection of airport ground handling equipment were determined by the expert AGHSEVS decision-making team. Professional experience was utilized by the team in the determination of important criteria. Explanations of important criteria and their definitions are provided in Table $4[4,6,10,36]$.

Table 4. Airport ground handling equipment evaluation criteria and its definitions.

\begin{tabular}{cc}
\hline Equipment Criteria & Definition of Importance \\
\hline$\left(E C_{1}\right)$ Equipment quality & Good equipment quality control \\
mean-time-between-failures (MTBF) \\
Good operation in airport work environment
\end{tabular}

Following the determination of criteria, AGHSEVS processes under development or in use were investigated, and the expert AGHSEVS decision-making team determined all criteria on the basis of the needs of the company. The six criteria were equipment quality $\left(E C_{1}\right)$, safety mechanisms $\left(E C_{2}\right)$, maintenance $\left(E C_{3}\right)$, technical transfer and mechanic training $\left(E C_{4}\right)$, quick supply after sales and technical services and cooperative relationship $\left(E C_{5}\right)$, and the reasonableness of the price $\left(E C_{6}\right)$. These criteria were used to develop the decision hierarchy, which was established accordingly. A decision hierarchy, structured by the team, and related criteria are displayed in Figure 3. 


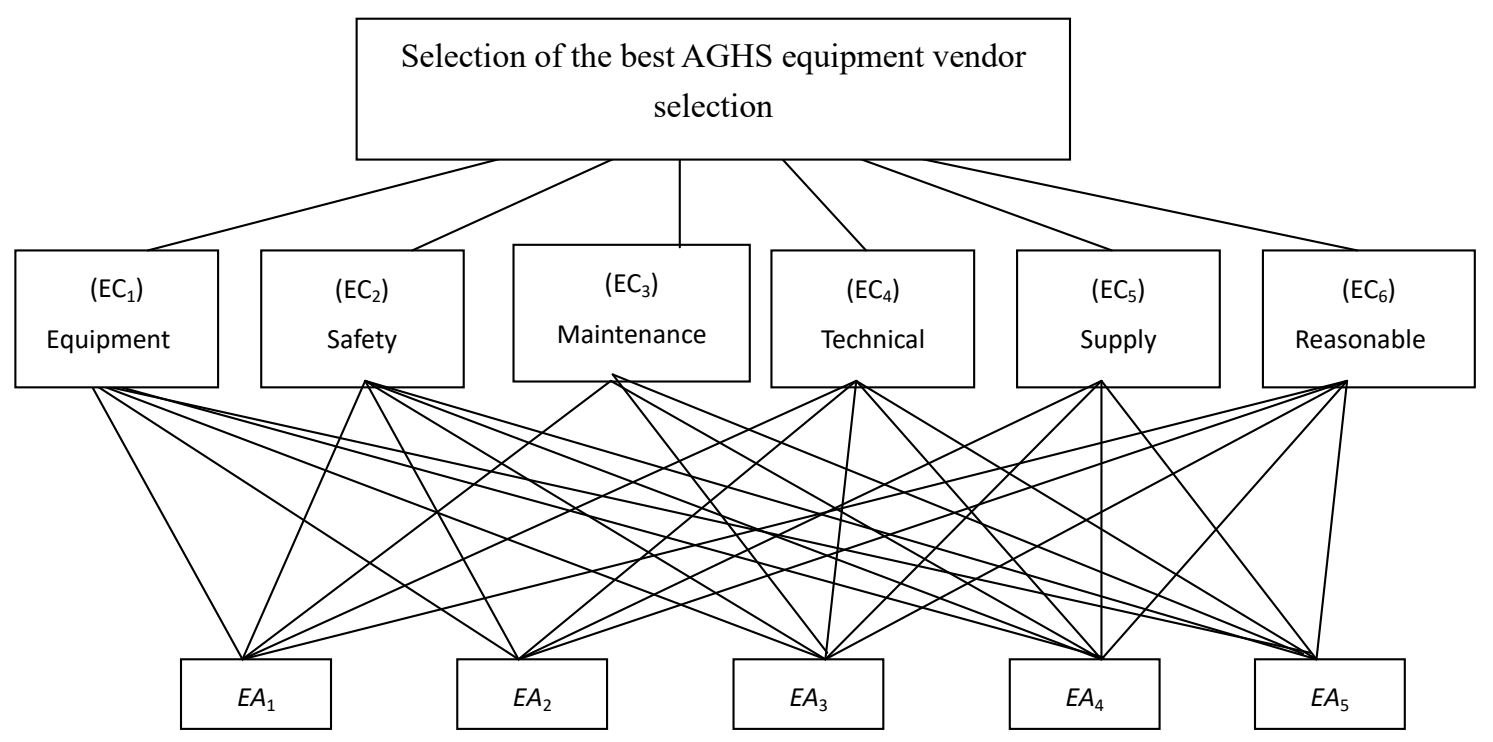

Figure 3. The decision hierarchy of AGHSEVS.

\subsection{Calculation of Criterion Weights}

After developing the decision hierarchy, criterion weights were computed using the AHP method. In this phase, experts on the team developed the individual pairwise comparison matrix; all scales used in the pairwise comparison matrix are provided in Table 1 . The geometric means of these values were determined to obtain the pairwise comparison matrix that achieved a consensus among team members (Table 5).

Table 5. The pairwise comparison matrix criteria.

\begin{tabular}{lcccccc}
\hline & $\boldsymbol{E} \boldsymbol{C}_{\mathbf{1}}$ & $\boldsymbol{E C _ { \mathbf { 2 } }}$ & $\boldsymbol{E C _ { \mathbf { 3 } }}$ & $\boldsymbol{E C _ { \mathbf { 4 } }}$ & $\boldsymbol{E C _ { \mathbf { 5 } }}$ & $\boldsymbol{E C _ { \mathbf { 6 } }}$ \\
\hline$E C_{1}$ & 1.0 & 0.2 & 0.3 & 0.4 & 0.2 & 0.6 \\
$E C_{2}$ & 0.5 & 1.0 & 3.4 & 1.9 & 2.3 & 2.3 \\
$E C_{3}$ & 2.4 & 0.4 & 1.0 & 0.3 & 0.4 & 0.4 \\
$E C_{4}$ & 0.4 & 0.5 & 3.2 & 1.0 & 1.5 & 2.4 \\
$E C_{5}$ & 1.9 & 0.4 & 2.1 & 0.5 & 1.0 & 1.9 \\
$E C_{6}$ & 1.8 & 2.9 & 2.3 & 0.3 & 2.8 & 1.0 \\
\hline
\end{tabular}

The outcomes (Table 6) were obtained from computations based on the pairwise comparison matrix (Table 4). EC $2, E C_{4}$ and $E C_{6}$ were determined as the most essential criteria for AGHSEVS, according to the AHP. The $C R$ of the pairwise comparison matrix was computed as $0.055<0.1$. Thus, the weights were shown to be consistent, and, consequently, they were used in the subsequent selection procedures.

Table 6. Results obtained with AHP.

\begin{tabular}{cccc}
\hline Equipment Criteria & Weights $(w)$ & $\lambda_{\max }, C I, R I$ & $C R$ \\
\hline$E C_{1}$ & 0.067 & $\lambda_{\max }=6.303$ & \\
$E C_{2}$ & 0.277 & & \\
$E C_{3}$ & 0.114 & $\mathrm{CI}=0.060$ & 0.050 \\
$E C_{4}$ & 0.195 & & \\
$E C_{5}$ & 0.167 & $\mathrm{RI}=1.24$ & \\
$E C_{6}$ & 0.179 & & \\
\hline
\end{tabular}




\subsection{Evaluation of Alternatives and Determining the Rank}

During Step 1, when alternatives were evaluated, team members were asked to establish the decision matrix by comparing alternatives under each criterion separately. A fuzzy evaluation matrix, established by evaluating the choice of ground handling equipment according to linguistic variables in Tables 4 and 7, was composed of triangular fuzzy numbers equivalent to linguistic variables. In Step 1, the fuzzy evaluation matrix was determined using the criterion weights computed through the AHP (Table 6). In Step 2, the weighted evaluation matrix was developed using Equation (4). The resulting fuzzy weighted decision matrix is displayed in Table 8. In Table 9, the elements $v_{i j}, \forall i, j$ are normalized by positive triangular fuzzy numbers and their scopes belong to a closed interval [0,1]. For AGHSEVS, we used the aforementioned definition of the fuzzy positive-ideal solution and fuzzy negative-ideal solution as $\widetilde{v}_{i}^{+}=(1,1,1)$ and $v_{i}^{-}=(0,0,0)$ for the benefit criterion, and $\widetilde{v}_{i}^{+}=(0,0,0)$ and $v_{i}^{-}=(1,1,1)$ for the cost criterion. Step 3, Equations (5) and (6). For AGHSEVS, $E C_{2}$ and $E C_{6}$ were cost criteria, whereas the remaining criteria were benefit criteria. In Step 3, the distance of each alternative from $D^{+}$and $D^{-}$could be calculated using Equations (7) and (8). Step 4 involved identifying an ideal solution according to the similarity by using Equation (9). Detailed illustrations of the method are provided by Dağdeviren et al. [10]. Similar calculations were conducted for the other alternatives, and the outcome of the fuzzy TOPSIS method is provided in Table 8 . Based on $C C_{j}$ values, the ranking of the alternatives in descending order is $E A_{3}, E A_{1}, E A_{2}, E A_{5}$ and $E A_{4}$. The results of the proposed model indicated that $E A_{3}$ is the most suitable alternative, with a $C C$ value of 0.370 . The criteria have equal priorities in this case, and the $C C_{j}$ values obtained are presented in Table 10.

Table 7. Fuzzy evaluation matrix for the selection airport ground handling equipment.

\begin{tabular}{ccccccc}
\hline & $\boldsymbol{E} \boldsymbol{C}_{\mathbf{1}}$ & $\boldsymbol{E C _ { \mathbf { 2 } }}$ & $\boldsymbol{E C _ { \mathbf { 3 } }}$ & $\boldsymbol{E C _ { \mathbf { 4 } }}$ & $\boldsymbol{E C _ { \mathbf { 5 } }}$ & $\boldsymbol{E C _ { \mathbf { 6 } }}$ \\
\hline$A_{1}$ & Excellent & Low & Medium & Very high & Medium & High \\
$A_{2}$ & High & Medium & Very high & High & High & Very high \\
$A_{3}$ & Very high & Medium & High & Excellent & High & Medium \\
$A_{4}$ & Low & Very high & Excellent & Medium & Medium & High \\
$A_{5}$ & Very high & High & Low & Very high & Very high & Excellent \\
$A_{1}$ & $(0.8,1,1)$ & $(0,0.2,0.4)$ & $(0.2,0.4,0.6)$ & $(0.6,0.8,1)$ & $(0.2,0.4,0.6)$ & $(0.4,0.6,0.8)$ \\
$A_{2}$ & $(0.4,0.6,0.8)$ & $(0.2,0.4,0.6)$ & $(0.6,0.8,1)$ & $(0.4,0.6,0.8)$ & $(0.4,0.6,0.8)$ & $(0.6,0.8,1)$ \\
$A_{3}$ & $(0.6,0.8,1)$ & $(0.2,0.4,0.6)$ & $(0.4,0.6,0.8)$ & $(0.8,1,1)$ & $(0.4,0.6,0.8)$ & $(0.2,0.4,0.6)$ \\
$A_{4}$ & $(0,0.2,0.4)$ & $(0.6,0.8,1)$ & $(0.8,1,1)$ & $(0.2,0.4,0.6)$ & $(0.2,0.4,0.6)$ & $(0.4,0.6,0.8)$ \\
$A_{5}$ & $(0.6,0.8,1)$ & $(0.4,0.6,0.8)$ & $(0,0.2,0.4)$ & $(0.6,0.8,1)$ & $(0.6,0.8,1)$ & $(0.8,1,1)$ \\
\hline$*$ Weight & 0.067 & 0.277 & 0.114 & 0.195 & 0.167 & 0.179 \\
\hline \multicolumn{7}{c}{ Note: ${ }^{*}$ AHP Weight. }
\end{tabular}

Table 8. Weighted evaluations for the alternative airport ground handling equipment.

\begin{tabular}{ccccccc}
\hline & $E C_{1}$ & $E C_{2}$ & $E C_{3}$ & $E C_{4}$ & $E C_{5}$ & $E C_{6}$ \\
\hline$A_{1}$ & $(0.054,0.067,0.067)$ & $(0.000,0.055,0.111)$ & $(0.023,0.046,0.068)$ & $(0.117,0.156,0.195)$ & $(0.033,0.067,0.100)$ & $(0.072,0.107,0.143)$ \\
$A_{2}$ & $(0.027,0.040,0.054)$ & $(0.055,0.111,0.166)$ & $(0.068,0.091,0.114)$ & $(0.078,0.117,0.156)$ & $(0.067,0.100,0.134)$ & $(0.107,0.143,0.179)$ \\
$A_{3}$ & $(0.040,0.054,0.067)$ & $(0.055,0.111,0.166)$ & $(0.046,0.068,0.091)$ & $(0.156,0.195,0.195)$ & $(0.067,0.100,0.134)$ & $(0.036,0.072,0.107)$ \\
$A_{4}$ & $(0.000,0.013,0.027)$ & $(0.166,0.222,0.277)$ & $(0.091,0.114,0.114)$ & $(0.039,0.078,0.117)$ & $(0.033,0.067,0.100)$ & $(0.072,0.107,0.143)$ \\
$A_{5}$ & $(0.040,0.054,0.067)$ & $(0.111,0.166,0.222)$ & $(0.000,0.023,0.046)$ & $(0.117,0.156,0.195)$ & $(0.100,0.134,0.167)$ & $(0.143,0.179,0.179)$ \\
$\mathrm{A}^{+}$ & $\widetilde{v}_{1}^{+}=(1,1,1)$ & $\widetilde{v}_{2}^{+}=(0,0,0)$ & $\widetilde{v}_{3}^{+}=(1,1,1)$ & $\widetilde{v}_{4}^{+}=(1,1,1)$ & $\widetilde{v}_{5}^{+}=(1,1,1)$ & $\widetilde{v}_{6}^{+}=(0,0,0)$ \\
$\mathrm{A}^{-}$ & $\widetilde{v}_{1}^{-}=(0,0,0)$ & $\widetilde{v}_{2}^{-}=(1,1,1)$ & $\widetilde{v}_{3}^{-}=(0,0,0)$ & $\widetilde{v}_{4}^{-}=(0,0,0)$ & $\widetilde{v}_{5}^{-}=(0,0,0)$ & $\widetilde{v}_{6}^{-}=(1,1.1)$ \\
\hline
\end{tabular}


Table 9. Fuzzy technique for order preference by similarity to ideal solution method results (weighted) $C C_{j}$.

\begin{tabular}{cccc}
\hline Alternatives & $\boldsymbol{D}^{+}$ & $\boldsymbol{D}^{-}$ & $\boldsymbol{C} \boldsymbol{C}_{\mathbf{j}}$ \\
\hline$E A_{1}$ & 3.852 & 2.182 & 0.362 \\
$E A_{2}$ & 3.919 & 2.108 & 0.350 \\
$E A_{3}$ & 3.793 & 2.232 & 0.370 \\
$E A_{4}$ & 4.075 & 1.953 & 0.324 \\
$E A_{5}$ & 3.975 & 2.048 & 0.340 \\
\hline
\end{tabular}

Table 10. Fuzzy TOPSIS weighted and unweight (i.e., no AHP weighted) rankings.

\begin{tabular}{ccccc}
\hline Rank & Weighted $C C_{j}$ & Weighted Ranking & Un-Weight $C C_{j}$ & Un-Weighted Ranking \\
\hline 1 & 0.370 & $E A_{3}$ & 0.669 & $E A_{3}$ \\
2 & 0.362 & $E A_{1}$ & 0.608 & $E A_{1}$ \\
3 & 0.350 & $E A_{2}$ & 0.560 & $E A_{2}$ \\
4 & 0.340 & $E A_{5}$ & 0.508 & $E A_{5}$ \\
5 & 0.324 & $E A_{4}$ & 0.432 & $E A_{4}$ \\
\hline
\end{tabular}

Based on un-weighted $C C_{j}$ values, the ranking of the alternatives in descending order is $E A_{3}$, $E A_{1}, E A_{2}, E A_{5}$ and $E A_{4}$. The most suitable alternative is the same as that for the weighted ranking. A detailed discussion of fuzzy TOPSIS methods is provided by Dağdeviren et al. [10].

For validation, a comparing was performed with the FWA left and right scores, and the ranking is $E A_{3}, E A_{5}, E A_{1}, E A_{2}$ and $E A_{4}$. These results are similar to the fuzzy TOPSIS results ( $E A_{3}, E A_{1}, E A_{2}, E A_{5}$ and $E A_{4} ;$ Table 11).

Table 11. Fuzzy average weighted left and right scores rank result.

\begin{tabular}{ccccc}
\hline Vendor Number & $\boldsymbol{L}$ Value & $\boldsymbol{R}$ Value & FWA Average Value & Rank \\
\hline$E A_{1}$ & 0.264 & 0.587 & 0.425 & 3 \\
$E A_{2}$ & 0.318 & 0.426 & 0.372 & 4 \\
$E A_{3}$ & 0.318 & 0.593 & 0.456 & 1 \\
$E A_{4}$ & 0.318 & 0.418 & 0.368 & 5 \\
$E A_{5}$ & 0.377 & 0.474 & 0.426 & 2 \\
\hline
\end{tabular}

\subsection{Comparing Solutions for the LP, MCGP and MAGP Models}

The LP, MCGP, and MAGP models were solved using the Lingo 11.0 software package. For the LP model, the ideal vendor and its optimal quantities were calculated as: $\left(g_{1}\right)$ TVP $=12.17,\left(g_{2}\right) f_{2}=$ $288,\left(g_{3}\right) f_{3}=1.84,\left(g_{4}\right) f_{4}=33.22, X_{1}=15, X_{2}=0, X_{3}=18, X_{4}=0$, and $X_{5}=0$. For the MCGP model, the ideal vendor and its optimal quantities were calculated as: $\left(g_{1}\right)$ TVP $=12.16,\left(g_{2}\right) f_{2}=288,\left(g_{3}\right) f_{3}$ $=1.80,\left(g_{4}\right) f_{4}=33.33, X_{1}=15, X_{2}=3, X_{3}=16, X_{4}=0$, and $X_{5}=0$. For the MAGP model, the ideal vendor and its optimal quantities were calculated as $\left(g_{1}\right)$ TVP $=11.69,\left(g_{2}\right) f_{2}=276,\left(g_{3}\right) f_{3}=1.77,\left(g_{4}\right)$ $f_{4}=31.93, X_{1}=15, X_{2}=0, X_{3}=17, X_{4}=0$, and $X_{5}=0$. This indicated that $X_{3}\left(E A_{3}\right)$ is the ideal vendor with the maximum purchase quantity.

\section{Conclusions and Implications}

\subsection{Conclusions and Limitations}

Finally, for verifying an LP model, we employed multi-choice goal programming (MCGP) and multi-aspiration goal programming (MAGP) to perform comparative analyses. The proposed MCGP approaches therefore could improve the practical applications of AGHSEVS model. In addition, this allows DMs to solve AGHSEVS problems when considering their preferences. 


\section{Limitations}

In order to eliminate the fuzzy TOPSIS method based on FWA left and right score methods, drawback and achieve the accurate results, we are comparing two GP to verify result of the AGHSEVS LP model. Otherwise, If DMs use the new fuzzy TOPSIS method [37], the conjunction GP approach [38] can be a different result.

\subsection{Management Implications}

Our AGHSEVS model provides four contributions: (i) the AGHSEVS model can help DMs identify appropriate AGHS equipment and enable purchasing managers to easily select the ideal AGHSEVS on the basis of criteria such as equipment quality, technical transfer and mechanic training, and reasonableness of the price; (ii) the systematic new evaluation procedure can help AGHS company managers make optimal decisions for AGHSEVS; (iii) the AGHSEVS model successfully solves the AGHSEVS problem by considering both qualitative and quantitative methods; and (iv) the model can be used, with slight modifications, in other decision-making problems in AGHS companies and in other countries.

\subsection{Future Directions}

For future studies, other multi-criteria methods, such as the fuzzy PROMETHEE and ELECTRE methods, can be used to solve AGHSEVS problems. Additionally, other mathematical models, such as the AHP-QFD model or DEA model [39,40], can be combined with the model proposed here. This can potentially improve our proposed model, which is the goal of our future research.

Author Contributions: Conceptualization, Y.-T.P.; formal analysis, C.-S.T. and Y.-T.P.; writing-original draft preparation, C.-S.T. and Y.-T.P.; writing-review and editing, C.-S.T. and Y.-T.P.; planning all works in the study and supervision, C.-W.S.

Funding: This research received no external funding.

Conflicts of Interest: The authors declare no conflict of interest.

\section{References}

1. Fuhr, J.; Beckers, T. Vertical governance between airlines and airport: A transaction cost analysis. Rev. Netw. Econ. 2006, 5, 386-412. [CrossRef]

2. Schmidberger, S.; Bals, L.; Hartmann, E.; Jahns, C. Ground handling services at European hub airports: Development of a performance measurement system for benchmarking. Int. J. Prod. Econ. 2009, 117, 104-111. [CrossRef]

3. Kazda, A.; Caves, R.E. Airport Design and Operations, 1st ed.; Emerald Group Publishing Limited: London, UK, 2000.

4. Bard, J.F.; Sousk, S.F. A tradeoff analysis for rough terrain cargo handlers using the AHP: An example of group decision making. IEEE Trans. Eng. Manag. 1990, 37, 222-228. [CrossRef]

5. Wang, T.C.; Chang, T.H. Application of TOPSIS in evaluating initial training aircraft under a fuzzy environment. Expert Syst. Appl. 2007, 33, 870-880. [CrossRef]

6. Byun, D.H. The AHP approach for selecting an automobile purchase model. Inf. Manag. 2001, 38, $289-297$. [CrossRef]

7. Shang, J.; Sueyoshi, T. A unified framework for the selection of a flexible manufacturing system. Eur. J. Oper. Res. 1995, 85, 297-315. [CrossRef]

8. Lin, Z.C.; Yang, C.B. Evaluation of machine selection by the AHP method. J. Mater. Process. Technol. 1996, 57, 253-258. [CrossRef]

9. Chan, F.T.S.; Ip, R.W.L.; Lau, H. Integration of expert system with analytic hierarchy process for the design of material handling equipment selection system. J. Mater. Process. Technol. 2001, 116, 137-145. [CrossRef]

10. Dağdeviren, M.; Yavuz, S.; Kılınç, N. Weapon selection using the AHP and TOPSIS methods under fuzzy environment. Expert Syst. Appl. 2009, 36, 8143-8151. [CrossRef] 
11. Shipley, M.F.; Korvin, A.D.; Obid, R. A decision making model for multi-attribute problems incorporating uncertainty and bias measures. Comput. Oper. Res. 1991, 18, 335-342. [CrossRef]

12. Shyur, H.J.; Shih, H.S. A hybrid MCDM model for strategic vendor selection. Math. Comput. Model. 2006, 44, 749-761. [CrossRef]

13. Chang, C.T. Multi-choice goal programming. Omega 2007, 35, 389-396. [CrossRef]

14. Vratb, K.R.P.; Kumarc, P. A goal programming model for paper recycling system. Omega 2008, 36, $405-417$.

15. Saaty, T.L. The Analytic Hierarchy Process; McGraw-Hill: New York, NY, USA, 1980.

16. Kulak, O.; Durmuşoğlu, M.B.; Kahraman, C. Fuzzy multi-attribute equipment selection based on information axiom. J. Mater. Process. Technol. 2005, 169, 337-345. [CrossRef]

17. Chan, F.T.; Kumar, N. Global supplier development considering risk factors using fuzzy extended AHP-based approach. Omega 2007, 35, 417-431. [CrossRef]

18. Wang, J.J.; Yang, D.L. Using a hybrid multi-criteria decision aid method for information systems outsourcing. Comput. Oper. Res. 2007, 34, 3691-3700. [CrossRef]

19. Hwang, C.L.; Yoon, K. Multiple Attribute Decision Making: Methods and Applications, A State of the Art Survey; Springer: New York, NY, USA, 1981.

20. Wang, Y.J. Applying FMCDM to evaluate financial performance of domestic airlines in Taiwan. Expert Syst. Appl. 2008, 34, 1837-1845. [CrossRef]

21. Chen, C.T. Extensions of the TOPSIS for group decision-making under fuzzy environment. Fuzzy Sets Syst. 2000, 114, 1-9. [CrossRef]

22. Chu, T.C.; Lin, Y.C. Improved extensions of the TOPSIS for group decision-making under fuzzy environment. J. Inf. Optim. Sci. 2002, 23, 273-286.

23. Lai, Y.J.; Hawang, C.L. Fuzzy Multiple Objective Decision Making, Methods and Applications; Springer: Berlin/Heidelberg, Germany, 1994.

24. Ding, J.F.; Liang, G.S. Using fuzzy MCDM to select partners of strategic alliances for liner shipping. Inf. Sci. 2005, 173, 197-225. [CrossRef]

25. Zadeh, L.A. Fuzzy sets. Inf. Control 1965, 8, 338-353. [CrossRef]

26. Karsak, E.E.; Tolga, E. Fuzzy multi-criteria decision-making procedure for evaluating advanced manufacturing system investments. Int. J. Prod. Res. 2001, 69, 49-64. [CrossRef]

27. Xu, Z.S.; Chen, J. An interactive method for fuzzy multiple attribute group decision making. Inf. Sci. 2007, 177, 248-263. [CrossRef]

28. Chen, C.T.; Lin, C.T.; Huang, S.F. A fuzzy approach for supplier evaluation and selection in supply chain management. Int. J. Prod. Econ. 2006, 102, 289-301. [CrossRef]

29. Önüt, S.; Soner, S. Transshipment site selection using the AHP and TOPSIS approaches under fuzzy environment. Waste Manag. 2008, 28, 1552-1559. [CrossRef] [PubMed]

30. Mokhtarian, M.N. A new fuzzy weighted average (FWA) method based on left and right scores: An application for determining a suitable location for gas oil station. Comput. Math. Appl. 2011, 61, 3136-3145. [CrossRef]

31. Guneri, A.F.; Yucel, A.; Ayyildiz, G. An integrated fuzzy-lp approach for a supplier selection problem in supply chain management. Expert Syst. Appl. 2009, 36, 9223-9228. [CrossRef]

32. Charnes, A.; Cooper, W.W.; Ferguson, R. Optimal estimation of executive compensation by linear programming. Manag. Sci. 1955, 1, 138-151. [CrossRef]

33. Lin, C.T.; Huang, S.F. A Fuzzy Approach for Supplier Evaluation and Selection in Supply Chain Management; Da-Yeh University: Changhua, Taiwan, 2004; submitted to International Journal of Production Economics.

34. Chang, Y.H.; Wey, W.M.; Tseng, H.Y. Using ANP priorities with goal programming for revitalization strategies in historic transport: A case study of the Alishan Forest Railway. Expert Syst. Appl. 2009, 36, 8682-8690. [CrossRef]

35. Chang, C.T. Revised multi-choice goal programming. Appl. Math. Model. 2008, 32, 2587-2595. [CrossRef]

36. Hossein, K.; Attarpour, M. Multi-aspiration goal programming formulation. Int. J. Ind. Eng. 2012, 19, 456-463.

37. Relich, M.; Pawlewski, P. A fuzzy weighted average approach for selecting portfolio of new product development projects. Neurocomputing 2017, 231, 19-27. [CrossRef]

38. Chung, C.K.; Chen, H.M.; Chang, C.T.; Huang, H.L. On fuzzy multiple objective linear programming problems. Expert Syst. Appl. 2018, 114, 552-562. [CrossRef] 
39. Tu, C.S.; Chang, C.T.; Chen, K.K.; Lu, H.A. Applying an AHP-QFD Conceptual Model and Zero-One Goal Programming to Requirement-Based Site Selection for an Airport Cargo Logistics Center. Int. J. Inf. Manag. Sci. 2010, 21, 407-430.

40. Ferreira, D.C.; Marques, R.C.; Pedro, M.I. Comparing efficiency of holding business model and individual management model of airports. J. Air Trans. Manag. 2016, 57, 168-183. [CrossRef]

(C) 2019 by the authors. Licensee MDPI, Basel, Switzerland. This article is an open access article distributed under the terms and conditions of the Creative Commons Attribution (CC BY) license (http://creativecommons.org/licenses/by/4.0/). 